\title{
Submillimeter corrections to gravity and the metastability of white dwarf and neutron stars
}

\author{
Mofazzal Azam ${ }^{1}$ and M. Sami ${ }^{2}$ \\ ${ }^{1}$ Theoretical Physics Division, \\ Bhabha Atomic Research Centre \\ Mumbai, India \\ ${ }^{2}$ Department of physics, \\ Jamia Millia Islamia, New Delhi.
}

\begin{abstract}
The string inspired higher dimensional theories suggest modification of Newton's law at submillimeter length scales. Inter-particle distances in white dwarf and neutron stars are $10^{-10} \mathrm{cms}$ and $10^{-13} \mathrm{cms}$ respectively, and therefore, the effects of of short distance corrections to gravity deserve investigation. We show, by carrying out explicit analytical many-body calculations that, in the presence of corrections, the normal state of these compact stars become metastable. The actual quantum mechanical ground state of the stars turns out to be unstable. However, the tunneling probability to the unstable ground state is so small that the stars may remain trapped in the metastable state practically for ever.
\end{abstract}

PACS numbers: $04.50 .+\mathrm{h}$

\section{INTRODUCTION}

The brane-world scenario proposed by Randall and Sundrum 1, 2], gives rise to modification of gravity at submillimeter distance scales; the corrections are in higher powers of inverse distance and appear as additional terms in the Newtonian potential. The modified potential is given by,

$$
\Phi(r)=-\frac{G M}{r}\left(1+\frac{2 l^{2}}{3 r^{2}}\right)
$$

where $l$ is the curvature scale of 5-dimensional antideSitter space time, and as before, $G$ is the usual Newton's constant of gravity, $M$ is the mass, $r$ is the distance in 3-space. In the setting above, $l$ has the dimension of length in 3-space. Henceforth, we will take $l_{s}^{2}=\frac{2}{3} l^{2}$.

The astrophysical consequences of short distance modifications of gravity are discussed in Refs. 3, 44]. The average inter-particle distance in compact stars such as the white dwarf and neutron stars are $10^{-10} \mathrm{cms}$ and $10^{-13} \mathrm{cms}$ respectively, and therefore, the submillimeter corrections, in principle, would effect such parameters as mass and radius, and also the mass-radius relationship of the stars. Wiseman [4] has carried out a detailed numerical study of the neutron stars on the brane and has claimed that these parameters of the neutron stars are not effected by brane world corrections.

In Ref. [5], we have carried out some simple analytical many-body calculations using the brane modified gravitational potential. Our approach was based on manybody theory in semi-relativistic quantum mechanics. It was demonstrated that the inclusion of the brane correction terms does not effect the Chandrasekhar ground state which is consistent with the results obtained by Wiseman using numerical technique [4]. In this paper, we intend to show that, although correction terms do not effect the variational minimum of the energy corresponding to Chandrasekhar ground state, they give rise to two new features of energy as a function of the radius: a positive maximum very close to the origin and a new unbounded minimum at the origin itself. The unbounded minimum indicates the absence of true quantum mechanical ground state and, there by, a quantum mechanical instability. This implies that the normal Chandrasekhar ground state of these compact stars become metastable. We show that the tunneling probability of the stars from the metastable state to the unstable ground state is vanishingly small.

\section{THE KINETIC ENERGY OF RELATIVISTIC DEGENERATE ELECTRON GAS}

We consider quantum mechanics of a self-gravitating system of large number of fermions in a compact spherical volume, $V$, with a large constant density such that fermions are relativistic. We will, first, consider here the white dwarf stars and, then, argue that our results can also be carried over to neutron stars. Consider a white dwarf star whose constituents are atoms of mass number $A$ and atomic number $Z$. Let the number of atoms in the star be $N_{A}$. The star is charge neutral, and therefore, the total number of electrons in star, $N_{e}=Z N_{A}$ and the total number of nucleons (protons+neutrons) $N_{n}=A N_{A}$. Latter, we will see that, $N_{e} / N_{n}=Z / A=Y_{e}$ is an important characteristic of a white dwarf star. Our basic assumption are:

(1) For white dwarf stars general relativistic effects can be neglected.

(1) Atomic nuclei are practically motionless and provide the entire gravitational attraction.

(2) The star is so dense that electrons constitute a degenerate relativistic Fermi gas at zero temperature.

The kinetic energy of the star is the energy of the relativistic degenerate free Fermi gas which can be evaluated as follows 7]. The single-particle electron states are spec- 
ified by the momentum $\mathbf{p}$ and the spin quantum numbers $s= \pm 1 / 2$. The single-particle energy levels are independent of $s$ :

$$
\epsilon_{\mathbf{p} s}=\sqrt{(\mathbf{p} c)^{2}+\left(m_{e} c^{2}\right)^{2}}-m_{e} c^{2}
$$

where $m_{e}$ is the electron mass. The energy of free Fermi electron gas is given by,

$\mathcal{E}_{F}=\mathcal{E}_{0} \overline{\mathcal{E}}\left(x_{F}\right)-N_{e} m_{e} c^{2} ; \quad \mathcal{E}_{0}=\frac{\left(m_{e} c^{2}\right)^{4}}{(c \hbar)^{3}} V ;$

$\overline{\mathcal{E}}\left(x_{F}\right)=\frac{1}{8 \pi^{2}}\left[x_{F}\left(1+2 x_{F}^{2}\right) \sqrt{1+x_{F}^{2}}-\ln \left(x_{F}+\sqrt{1+x_{F}^{2}}\right)\right]$

where $x_{F}=p_{F} / m_{e} c$ and the Fermi momentum is given by $p_{F}=\hbar\left(3 \pi^{2} n_{e}\right)^{1 / 3}\left(n_{e}=N_{e} / V\right)$.

In terms of $x_{F}, \mathcal{E}_{0}=3 \pi^{2} N_{e} m_{e} c^{2} / x_{F}^{3}$. Therefore, the kinetic energy of the electron gas for constant electron density is given by

$$
K . E . \equiv \mathcal{E}_{F}=\frac{3 \pi^{2} N_{e} m_{e} c^{2}}{x_{F}^{3}} \overline{\mathcal{E}}\left(x_{F}\right)-N_{e} m_{e} c^{2}
$$

For variable electron density, a similar expression has been derived by Daubechies in a very general and mathematically rigorous way ([8], see also [9]). We will mainly be considering spherically symmetric stars with a constant density profile. Following reference [10], we express the dimensionless Fermi momentum, $x_{F}$, in terms of mass and radius of the white dwarf stars as follows. Let, $M_{0}$ and $R_{0}$, of dimensions of mass and length be given by,

$$
\begin{aligned}
& M_{0}=\frac{5}{6} \sqrt{15 \pi}\left(\frac{c \hbar}{G m_{n}^{2}}\right)^{3 / 2} m_{n} Y_{e}^{2}=10.599 M_{\odot} Y_{e}^{2} \\
& R_{0}=\frac{\sqrt{15 \pi}}{2}\left(\frac{c \hbar}{G m_{n}^{2}}\right)^{1 / 2} \frac{c \hbar}{m_{e} c^{2}} Y_{e}=17250 k m \times Y_{e}
\end{aligned}
$$

For helium white dwarf stars, $Y_{e}=1 / 2$, therefore, $M_{0}=$ $2.650 M_{\odot}$ and $R_{0}=8623 \mathrm{~km}$.

It will also be convenient to introduce the dimensionless mass and radius of stars as $\bar{M} \equiv M / M_{0}$ and $\bar{R} \equiv R / R_{0}$ in terms of which $x_{F}=\bar{M}^{1 / 3} / \bar{R}$.

\section{THE INTERACTION POTENTIALS AND THE POTENTIAL ENERGY}

We consider a spherically symmetric star of mass $M$ and radius $R$, with a constant mass density profile $\rho_{A}(r)=\rho=$ constant for $0 \leq r \leq R$, and $\rho_{A}(r)=0$ for $r>R$. Therefore, $M=\bar{N}_{A} m_{A}=\frac{4 \pi}{3} \rho R^{3}$. In what follows we briefly describe the potential energy of the system in presence of short range corrections due to RandallSundrum (RS) and Yukawa corrections (see Ref. [5] for details) At any point $\vec{r}$ inside a solid sphere, the potential consists of two parts: one part is due to the inner sphere bellow the point $\vec{r}$ and second part is due to the outer spherical shell. Let $\Phi^{(1)}(r, a)$ be the potential outside a solid sphere of radius $a$, and $\Phi^{(2)}(r, b, R)$ be the potential inside a spherical shell of inner radius $b$ and outer radius $R$. The potential energy is given by,

$U=\frac{1}{2}\left[\int_{0}^{R} 4 \pi \rho r^{2} d r\left(\Phi^{(1)}(r-\epsilon, r)+\Phi^{(2)}(r, r+\epsilon, R)\right)\right]$

At the end of the calculations, we try to take the limit $\epsilon \rightarrow 0$. If the limit does not exist, we take $\epsilon$ to be the smallest inter-particle distance.

Using the expressions for the potentials $\Phi_{R S}^{(1)}(r, a)$ and $\Phi_{R S}^{(2)}(r, b, R)$ obtained in Ref. [5], we obtain the potential energy corresponding to RS corrections in Eq. (1),

$$
U_{R S}=-\frac{3}{2} \frac{G M^{2} l_{s}^{2}}{R^{3}} \ln \frac{R}{\epsilon}
$$

Here $\epsilon$ is the average inter-particle distance in the star. For a system of Fermions, it will be proportional to $R / N^{1 / 3}$. The precise numerical factor which is of order one, will not matter for our discussions, and therefore, we will simply take $\epsilon=R / N_{n}^{1 / 3}$. The potential energy can, therefore, be written as

$$
U_{R S}=-\frac{3}{2} \frac{G M^{2} l_{s}^{2}}{R^{3}} \ln \left(N_{n}^{1 / 3}\right)
$$

In terms of the variables $M_{0}$ and $R_{0}$,

$$
U_{R S}=-\left(\frac{5 l_{s}^{2} \ln \left(N_{n}^{1 / 3}\right)}{2 R_{0}^{2}}\right)\left(\frac{3}{5} \frac{G M^{2}}{R}\right)\left(\frac{R_{0}^{2}}{R^{2}}\right)
$$

Let us call the terms in the first bracket $\beta$. In terms of the Fermi momentum, $x_{F}$ the total potential energy corresponding brane modified potential given by Eq. (1) is

$$
U=-N_{e} m_{e} c^{2} \bar{M}^{2 / 3} x_{F}-N_{e} m_{e} c^{2} \beta x_{F}^{3}
$$

where the first part in the above equation is contributed by the Newtonian potential. For submillimeter corrections, $\beta \simeq 10^{-19}$ for helium stars. For other stars it varies within a order magnitude of this number.

Yukawa interaction is a very generic short range interaction (see 11] for details) and therefore, the results obtained for this potential is expected to hold qualitatively for most short range potentials. On phenomenological grounds we require, that the strength of the potential due to the correction term at the millimeter length scale, at the best, be comparable to gravity. This means that the parameter, $\alpha$, characterizing the strength of potential, be a number of the order of unity. We will keep in mind this fact while preserving $\alpha$ in all our calculations [12].

For spherically symmetric star of radius $R$ and constant mass density $\rho$, the many-body potential at any 
point $r, 0 \leq r \leq R$ is given by [1],

$$
\Phi_{Y}(r)=-\frac{4 \pi G \alpha \rho}{\mu^{2}}\left(1-\left[\left(R+\frac{1}{\mu}\right) e^{-\mu R} \frac{\sinh (\mu r)}{r}\right]\right)
$$

Let us note that the second term in the bracket is very small and is negligible in the body of the star but it is large close to the boundary and will give rise to surface tension as in the case of Randall-Sundrum corrections. We are interested in the balance of pressure and gravity (along with various corrections) in the body of the star, and therefore, we neglect this term. Thus in the potential given in Eq.(8), the only relevant part is: $\Phi_{Y}(r)=-4 \pi G \alpha \rho / \mu^{2}$. Therefore, the potential energy,

$$
U_{Y}=\int_{0}^{R} 4 \pi r^{2} \rho \Phi_{Y}(r) d r=-N_{e} m_{e} c^{2} \gamma x_{F}^{3}
$$

where $\gamma=\left(\frac{5 \alpha}{\mu^{2} R_{0}^{2}}\right)$. For submillimeter corrections $\gamma \simeq$ $10^{-19}$ for helium stars. Note that the form of potential energy for both RS and Yukawa corrections are cubic in $x_{F}$. The dimensionless parameters $\beta$ (for RS) and $\gamma$ (for Yukawa) are of same order of magnitude and extremely small. Henceforth, we use $\beta$ for both.

Now, the relativistic quantum mechanical total energy is, $E\left(\bar{M}, x_{F}\right)=N_{e} m_{e} c^{2} \Xi\left(\bar{M}, x_{F}\right)$, where

$$
\Xi\left(\bar{M}, x_{F}\right)=\left[\frac{3 \pi^{2} \overline{\mathcal{E}}\left(x_{F}\right)}{x_{F}^{3}}-1-\bar{M}^{2 / 3} x_{F}-\beta x_{F}^{3}\right]
$$

In Eq.(10), the first two terms arise from the kinetic energy given in Eq.(2), the third term is due to the Newtonian potential energy (see reference [10]), and the last term comes, as shown in Eqs.(7) \& (9), from potential energy due to the short range corrections. In the absence of correction terms, the total energy is $E_{c}=$ $N_{e} m_{e} c^{2} \Xi_{c}\left(\bar{M}, x_{F}\right)$, where

$$
\Xi_{c}\left(\bar{M}, x_{F}\right)=\left[\frac{3 \pi^{2} \overline{\mathcal{E}}\left(x_{F}\right)}{x_{F}^{3}}-1-\bar{M}^{2 / 3} x_{F}\right]
$$

\section{GROUND STATE OF THE COMPACT STARS IN THE PRESENCE OF SHORT RANGE CORRECTION TO GRAVITY}

\section{A. The white dwarf stars}

Large quantum systems such as white dwarf stars are stable if the ground state energy per particle is bounded bellow. For a fixed value of $\bar{M}$, we look for the extrema of per-particle energy, $\Xi=E /\left(N_{e} m_{e} c^{2}\right)$, as a function of $x_{F}$. In the absence of corrections, it is known from the works of Chandrasekhar ([6], see also [9]) that bellow some critical mass, $\bar{M}_{c}$, the energy functional, $\Xi_{c}$ given in
Eq.(11), has a unique minimum. As we change the mass, the location of the minimum changes. By definition, $x_{F}$ is inversely proportional to the radius, and therefore, the variation of the location of the minimum with the mass gives the mass-radius relationship of the star.

We choose $\bar{M}=\bar{M}_{1}(\simeq 0.25)$, such that the star is Chandrasekhar stable with energy minimum at $x_{F}=\bar{x}_{1}(\simeq$ 0.8 ). Now consider the energy with correction terms, Eq.(7), for $\bar{M}=\bar{M}_{1}$. The correction term is too small, and become relevant only for very very large values of $x_{F}$ and therefore, the local minimum, which exist for small $x_{F}$ in the absence of corrections, will persist in the presence of the correction terms. Therefore, as before, energy as function of $x_{F}$ will have a local minimum at $x_{F}=\bar{x}_{1}$. However, for very large value of $x_{F}$, the energy approaches $-\infty$. To see this let us note, that for $x_{F}>>1, \frac{3 \pi^{2} \mathcal{E}\left(x_{F}\right)}{x_{F}^{3}}=\frac{3}{4} x_{F}$. Therefore,

$$
\Xi=\left[\frac{3}{4}-\bar{M}_{1}^{2 / 3}\right] x_{F}-1-\beta x_{F}^{3}
$$

Since $\bar{M}_{1}<M_{c}$, the expression in the square bracket in eq.(12) is positive. It is easy to see that $\Xi$, given above has a maximum for $x_{F}=\sqrt{\frac{1}{2 \beta}\left(\frac{3}{4}-\bar{M}_{1}^{2 / 3}\right)}$. The value of the function $\Xi$ at the point of the maximum is very large. Beyond the maximum, $\Xi$ rolls down to $-\infty$. To summarize, the energy with correction term has the following structure: for small $x_{F}$, there is a minimum, then for very large value there is a maximum after which it approaches $-\infty$. The dimensionless Fermi momentum, $x_{F}$, is inversely proportional to the radius, $\mathrm{R}$. Therefore, energy as a function of $R$ has a minimum for some finite value of $R$, say $R=R_{1}$ corresponding to the Chandrasekhar state. In addition it has a maximum very close to $R=0$ (say, for $R=R_{2}$ ), followed by an unbounded minimum at the origin. This makes the Chandrasekhar state metastable. In the dimensionless unit that we introduced, $R_{1}$ is of order one, where as $R_{2} \sim \sqrt{\beta} \sim 10^{-9.5}$, therefore, $R_{1} / R_{2}>>1$.

The potential and force due to the short range correction terms are similar to those in nuclear physics except that the strength is much weaker and the range is much larger. Therefore, a reference to nuclear physics may unveil qualitatively the relevant physical features. Consider, the energy-per-particle without the Newtonian term, $E^{\prime}\left(\bar{M}, x_{F}\right)=N_{e} m_{e} c^{2} \Xi^{\prime}\left(\bar{M}, x_{F}\right)$, where

$$
\Xi^{\prime}\left(\bar{M}, x_{F}\right)=\left[\frac{3 \pi^{2} \overline{\mathcal{E}}\left(x_{F}\right)}{x_{F}^{3}}-1-\beta x_{F}^{3}\right]
$$

This function has a maximum and an unbounded minimum. There is no other minimum. This feature is reminiscent of heavy nuclei, with nucleons interacting via the Yukawa potential. With suitable interpretation of the parameters, Eq.(13), can, in fact, be carried over to nuclear physics for quantitative treatment of heavy nuclei. 
It is well known in nuclear physics that the zero-point kinetic energy whether relativistic or non-relativistic can not balance the attractive potential energy of the Yukawa interaction. The problem is solved by invoking a very short range hard core repulsive potential which provides a lower bound and removes the unbounded minimum. Without this hard core repulsive potential, heavy nuclei can not be stable.

In our case we do not have a repulsive hard core potential. But the inclusion of long range Newtonian potential in Eq.(13) makes it identical to Eq.(10), and therefore, creates, bellow a critical mass, a local minimum in the energy function without effecting the other two features: a maximum and an unbounded minimum. The original Chandrasekhar ground state shows up as a metastable state in the local minimum of the energy functional.

\section{B. The neutron stars}

The neutron stars can be analyzed exactly in the same way as the white dwarf stars except for the fact that for neutron stars the general relativistic effects would play some role. In what follows, we, first, neglect these effects and comment on them at the end of this section. The first thing that should be noted is that both the kinetic and the potential energy in the neutron stars are due to the neutrons alone. Therefore, all the formulae and equations which are valid for the white dwarf stars are also valid for the neutron with the following replacements: (electron density, $n_{e} \rightarrow$ neutron density, $n_{n}$ ), (electron mass $m_{e} \rightarrow$ neutron mass, $m_{n}$ ), (number of electrons, $N_{e} \rightarrow$ number of neutrons $\left.N_{n}\right)$, and $\left(Y_{e} \rightarrow 1\right)$. This leads to the change in the numerical value of the following quantity, $M_{0} \rightarrow M_{0}^{(n)}=10.599 M_{\odot}, R_{0} \rightarrow R_{0}^{(n)}=$ $9.40 \mathrm{~km}, \beta \rightarrow \beta^{(n)}=\simeq 10^{-13}, \gamma \rightarrow \gamma^{(n)}=\simeq 10^{-13}$. The ground state of the neutron stars can be found just as in the case of white dwarfs. We reach the similar conclusion that, in the presence of short range corrections, neutron stars are metastable.

The general relativistic effects in the compact neutron stars, in our opinion, influences the Chandrasekhar ground state and the values of parameters such as mass and radius that are associated with it. It also changes the critical mass for the stability of the star. However, it should be clear from the discussions in the earlier sections that the short range correction to Newton's law of gravity does not effect the critical mass for the stability of the stars given by Chandrasekhar limit. It also does not effect the mass, radius or mass-relationship when the star is in the metastable Chandrasekhar ground state. This suggests that our results, in all likely hood, are valid in the presence of general relativistic effects.

\section{The probability of tunneling to the unstable ground state}

Let us provide an estimate of the probability of tunneling from the metastable state to the unstable ground state. It is obvious that the peak, at the maximum of the energy functional, that separates the metastable state (local minimum) from the unstable ground state (global minimum), acts as an energy barrier for the tunneling process. Let us recall some of the formulae from quantum mechanics on barrier penetration. Suppose that potential between two points, $x_{1}$ and $x_{2}$ increases monotonically, the point, $x_{2}$, is the peak of the potential and the energy of the particle crossing the barrier equals to its' value at the lower end. Then, the transmission coefficient or the tunneling probability, $T \sim e^{-S / \hbar}$, where the action, $S=\int_{x_{1}}^{x_{2}} p . d x$, and $S / \hbar>>1$. For the compact stars, let us represent by $R_{1}$, the radius of the star in the metastable ground state and, by $R_{2}$, the radius of the star at the local maximum separating the unstable ground state. As argued in the earlier sections, $R_{1}>>R_{2}$. The typical momenta of individual particle in these states are, approximately, given by the Fermi momenta, $\hbar N^{1 / 3} / R_{1}$ and $\hbar N^{1 / 3} / R_{2}$. Tunneling of the compact stars through the energy barrier at the maximum of the energy functional amounts to the change in the radius of spheres, occupied by individual particles, from $R_{1} / N^{1 / 3}$ to $R_{2} / N^{1 / 3}$. This means that interparticle distances change from $R_{1} / N^{1 / 3}$ to $R_{2} / N^{1 / 3}$, and as a result, momenta of individual particles change from $\hbar N^{1 / 3} / R_{1}$ to $\hbar N^{1 / 3} / R_{2}$. We will first calculate probability for such a tunneling for a single particle. The action $S$, for this can be given by $S=\int_{x_{1}}^{x_{2}} p \cdot d x \approx \Delta p \Delta x=$ $\left(\hbar N^{1 / 3} / R_{2}-\hbar N^{1 / 3} / R_{1}\right)\left(R_{1} / N^{1 / 3}-R_{2} / N^{1 / 3}\right)$. The condition, $R_{1}>>R_{2}, \rightarrow S / \hbar=R_{1} / R_{2}$. Therefore, the tunneling probability for a particle, $T_{\text {single }} \sim e^{-S / \hbar} \simeq$ $e^{-R_{1} / R_{2}}$. When all the particle, simultaneously, undergo such transitions, the compact star will tunnel through the energy barrier. For a star with particle number, $N$, the tunneling probability is, $T \approx e^{-N\left(R_{1} / R_{2}\right)}$. For typical white dwarf and neutron stars, particle number, $N \sim 10^{57}$, which is a huge number, and therefore, the tunneling probability to the unstable ground state is extremely small, and therefore, negligible.

\section{CONCLUSIONS}

We have analysed in details the effects of short range corrections to gravity on the compact stellar objects such as the white dwarf and neutron stars. The corrections lead to two new effects. One of them is the emergence of surface tension which is the characteristic of short range interactions and has been discussed in detail in our previous publication [5]. The second effect is that the compact stars become metastable. We have shown that the tunneling probability to the unstable ground state is so small 
that the stars once trapped in the metastable state would remain there.

\section{ACKNOWLEDGEMENTS}

We thank Sergei Odintsov for useful comments.

[1] L.Randall and R.Sundrum, Phys.Rev.Lett. 83, 3370 (1999); Phys.Rev.Lett. 83,4690 (1999)

[2] T. Shiromizu, K. Maeda and M. Sasaki, Phys. Rev. D62,024012 (2000); M. Sasaki, T. Shiromizu and K. Maeda, Phys. Rev. D62,024008 (2000); Roy Maartens, Phys. Rev. D 62, (2000) 084023; J. Garriga and T. Tanaka, Phys. Rev. Lett. 84, 2778 (2000); S. Nojiri, S. D. Odintsov, and S. Zerbini, Phys. Rev. D 62064006 (2000); S. Nojiri and S.D. Odintsov, hep-th/0209066 Roy Maartens, Brane-world gravity, gr-qc/0312059

[3] Cristiano Germani and Roy Maartens, Phys. Rev. D 64, 124010 (2001).

[4] T. Wiseman, Phys. Rev. D65 124007(2002); hep-th/0111057

[5] M. Azam and M. Sami, Phys. Rev. D72 024024(2005) ; gr-qc/0502026
[6] S. Chandrasekhar, Nobel Lecture, The Nobel Foundation 1984, Published in Rev. Mod. Phys. 56 (1984) 137

[7] Kerson Huang, Statistical Mechanics, 2nd Edition, John Wiley and Sons, 1987

[8] I. Daubechies, Commun. Math. Phys. 90(1983) 511

[9] E. H. Lieb and W. E. Thirring, Annales of Physics, 155 (1984) 494; E. H. Lieb and H-T. Yao, Commun. Math. Phys. 112 (1987) 147

[10] C. B. Jackson, J. Taruna, S. L. Pouliot, B. W. Ellison, D. D. Lee, and J. Piekarewicz, European Journal of Physics, 26 (2005) 695 ; astro-ph/0409348

[11] M. Azam and R. S. Gowda, nucl-th/0508030

[12] K. A. Bronnikov, S. A. Kononov, V. N. Melnikov, gr-qc/0601114 